\title{
Developments at ACM TODS
}

\author{
Richard Snodgrass \\ rtsecs.arizona.edu
}

TODS is in good shape. Submissions have been increasing steadily at about $5 \%$ each quarter, since statistics were started in July 2001. As the acceptance rate has been steady, that translates into more papers in the journal. (In doing so, quality was not compromised to increase quantity. We're simply getting more submissions, and so can accept more of the kind of paper we hope for.)

The December 2003 issue rounds up Volume 28 with twice as many papers as normal. We would need to go back to 1994 to find a volume of TODS with as many papers as the 2003 volume, and back to 1991 to find an issue with as many papers as the December 2003 issue. And 2004 looks even more promising. We're about to close out the March 2004 issue with a similar number of papers, and already have two papers accepted for the June 2004 issue (accepted papers are available very quickly on the web at http: / / www . acm.org/tods/Upcoming.html).

The December 2003 issue contains papers on a variety of topics, including

- Accommodating user preferences in queries,

- Dynamic mining, in which the user can focus the mining process through constraints that can be dynamically changed,

- Using a nondeterministic finite automaton to improve the performance of structure-oriented XML filtering, even over nested path expressions, by an order of magnitude,

- Selectivity estimation and histograms for spatio-temporal queries (queries over objects moving through space, or queries moving through space, or both),

- Refresh policies for web crawlers that cache remote pages and poll the sources periodically, and

- A survey (the second in TODS; the first appeared in the September issue) on using indexes for similarity search.

This increase in submissions encouraged me to enlarge the TODS Editorial Board. I have just appointed four new Associate Editors, bringing the Editorial Board to sixteen. The new Associate Editors are as follows.

- Mary Fernandez works at the juncture of programming languages and database systems, in particular on domain-specific languages for data management problems, their formal semantics, techniques for their efficient implementation, and their interaction with general-purpose programming languages

- Richard Hull works in areas related to the convergence of data and services, including research on e-services, workflow, policy management, personalization, data integration, telecom applications, languages and theory.

- Donald Kossmann works on techniques to improve the development and performance of Web-based information systems, in particular, on new query operators, advanced query processing techniques, and techniques to optimize whole database applications.

- Jennifer Widom works or has recently worked in the areas of data streams, caching and replication, XML and semistructured data, temporal databases, and data warehousing.

All four have contributed significantly both to our understanding of database systems through their research, and to the database community through their past service. I'm absolutely delighted that they are willing to help TODS continue to improve. 
ACM and the TODS Editorial Board go to great lengths to ensure that submissions are reviewed fairly and objectively. Perhaps this is an appropriate time to review the extensive conflict of interest policy followed by the TODS Editorial Board. The objective of this policy (available at http://www . acm. org/tods /COI.html) is to remove even the appearance of any conflict of interest. There are two places where conflicts of interest may arise: selection of the Associate Editor who handles the submission, and selection by the Associate Editor of the referees who review the submission.

For papers not co-authored by a member of the Editorial Board, the Editor-in-Chief (EiC) takes into account the affiliation of each author as well as knowledge about research collaborations in assigning an Associate Editor (AE) for the paper. The AE is required to inform the EiC of any potential conflict-ofinterest concerns. I have assigned papers to another AE whenever I thought that there would be any question about even the appearance of a conflict of interest.

For papers co-authored by a current $\mathrm{AE}$, the $\mathrm{EiC}$ will allocate the paper to an $\mathrm{AE}$ to handle, as is the normal procedure. However, the identity of the AE handling the paper will not be revealed to the authors. Instead, the $\mathrm{EiC}$ will serve as an intermediator in all communications between the $\mathrm{AE}$ handling the submission and the authors. All other aspects of the handling of such submissions will follow normal procedure.

For papers co-authored by the current Editor-in-Chief, the situation is somewhat more complex. The policy (http: / / www.acm.org/pubs/conflict_of_interest.html) provides the protocol, originally designed by an EiC of ACM's Transactions on Information and System Security, which seems appropriate!

1. The EiC will submit the paper to an $\mathrm{AE}$ who is specifically designated for this purpose and explicitly identified in the web pages for that journal. The designated AE must have agreed to accept this responsibility and should not be a collaborator of the EiC or from the same organization as the EiC.

2. The AE designated in step 1 (say Alice) will not process the paper herself, but will hand it to another $\mathrm{AE}$ (say Bob) whose identity will not be disclosed to the EiC. Bob will obtain reviews and make all decisions regarding processing of the paper (such as reject, requires major revision and second review, conditional accept, accept, etc.) and will convey these decisions to the EiC by way of Alice. Alice will keep the identity of Bob anonymous from the $\mathrm{EiC}$, and Bob will keep the identity of the reviewers anonymous from Alice.

3. In case of guest edited special issues, such as based on papers invited from conferences, the guest editor will make the final decision directly but will anonymize all reviewer information in corresponding with the authors, including the EiC.

4. In order to avoid the appearance of impropriety, existing standards of acceptability must be rigorously applied when considering papers (co-)authored by EiCs. Papers which are marginal in any way should be rejected.

Similarly, the AE takes into account the affiliation of each author as well as knowledge about research collaborations in assigning reviewers for the paper. A similarly high standard of avoiding even the appearance of a conflict of interest is applied.

These sometimes involved mechanisms are in place to assure the reader and potential authors that the papers that appear in TODS have been rigorously and objectively reviewed, and that the editorial decision for every submission has been based solely on the technical merit of that submission. 